\title{
The coherent dynamics of photoexcited green fluorescent proteins
}

\author{
Riccardo A. G. Cinelli, Valentina Tozzini, Vittorio Pellegrini, and Fabio Beltram \\ Scuola Normale Superiore and Istituto Nazionale per la Fisica della Materia, Piazza dei Cavalieri \\ 7, I-56126 Pisa, Italy \\ Giulio Cerullo, Margherita Zavelani-Rossi, and Sandro De Silvestri \\ Istituto Nazionale per la Fisica della Materia and Centro di Elettronica Quantistica e \\ Strumentazione Elettronica, Dipartimento di Fisica, Politecnico di Milano, Piazza Leonardo da \\ Vinci 32, I-20133 Milano, Italy \\ Mudit Tyagi and Mauro Giacca \\ Molecular Medicine Laboratory, International Centre for Genetic Engineering and Biotechnology, \\ Padriciano 99, I-34012 Trieste, Italy
}

\begin{abstract}
The coherent dynamics of vibronic wave packets in the green fluorescent protein is reported. At room temperature the non-stationary dynamics following impulsive photoexcitation displays an oscillating optical transmissivity pattern with components at $67 \mathrm{fs}\left(497 \mathrm{~cm}^{-1}\right)$ and $59 \mathrm{fs}\left(593 \mathrm{~cm}^{-1}\right)$. Our results are complemented by $a b$ initio calculations of the vibrational spectrum of the chromophore. This analysis shows the interplay between the dynamics of the aminoacidic structure and the electronic excitation in the primary optical events of green fluorescent proteins.
\end{abstract}

PACS numbers: 87.15.He, 87.15.Aa, 87.14.Ee, 78.47.+p 
The green fluorescent protein (GFP) of the Aequorea victoria jellyfish has emerged in recent years as a unique fluorescent label in several biological studies [1]. GFP is a large intrinsically-fluorescent protein (238 amino acids) characterized by a cylinder-shaped threedimensional structure with a diameter of $24 \AA$ and a height of $42 \AA$ [2]. The chromophore, located at the center of the cylinder, is a photoexcitable green-light emitter autocatalytically generated by the post-translational modification of a 3-amino-acid sequence (Ser65-Tyr66Gly67) [3]. It consists of the hydroxybenzyl side chain of Tyr66 (phenolic ring) and the imidazolidinone ring formed by cyclization of the tripeptide (heterocyclic ring). The spectral characteristics of its emission and absorption bands have received a tremendous amount of attention [2 5] in an effort to understand GFP photophysics down to the single-molecule level [6] and design mutants with optical properties tailored to specific needs. To date there exists a large set of GFP mutants with absorption and emission bands ranging from the violet to the red part of the spectrum. Recently time-dependent analyses have shed light on internal photoconversion mechanisms [7,8]. However, a definitive microscopic model accounting for the optical properties of the chromophore and its blinking and photobleaching dynamics [50 is still missing, partly due to a lack of precise information on the electronic states involved and to the limited effort in theoretical modelling. Only in recent years, in fact, a few electronic-structure calculations of the GFP chromophore in different protonation states have been performed [9.10].

Few-optical-cycle laser pulses allow ultrafast spectroscopy with unprecedented temporal resolution and are opening new avenues in the study of optical properties of molecules [11]. Creation of non-stationary vibronic wave packets and their observation have already provided new insights into the microscopic mechanisms responsible for the optical activity of few simple physical and biophysical systems [12 15. Observation of coherent phenomena in a protein, however, still remains a challenge because of fast dephasing times. There exist only few reports on coherent dynamics in proteic systems whose functionality, however, is determined by non-proteic cofactors [14, 15].

In this Letter we demonstrate that GFP ultrafast response after femtosecond laser ex- 
citation is dominated by the coherent dynamics of single-electron vibronic wave packets, created in both the ground and excited states of the protein chromophore [16]. The coherent dynamics manifests as an oscillatory modulation of the differential optical transmissivity. These results provide direct measurements of the chromophore collective vibrations during the optical process. In order to elucidate this dynamics, we also report the first ab initio calculations of the vibrational properties of the GFP chromophore based on the density functional theory (DFT).

The time-resolved technique here reported employed a pump-probe experimental scheme [13 with identical pump and probe pulses, resonant with GFP absorption. Sub-10 fs pulses centered around $500 \mathrm{~nm}$ were generated using a non-collinear optical parametric amplifier in the visible, pumped by the second harmonic of a Ti:sapphire laser [17]. The pulses had energies of about $40 \mathrm{~nJ}$ and spectral width allowing analysis in a broad wavelength range (490-550 nm). Samples consisted of GFP solutions at a concentration of $350 \mu \mathrm{M}$ in volumes of $40 \mu \mathrm{l} \mathrm{kept} \mathrm{in} \mathrm{a} 0.5 \mathrm{~mm}$-thick cuvette. Additionally, room-temperature steady-state absorption and emission spectra were measured. In particular, absorption was measured in a $300 \mu \mathrm{l} \mathrm{GFP} \mathrm{sample} \mathrm{at} \mathrm{a} \mathrm{concentration} \mathrm{of} 70 \mu \mathrm{M}$ in a $1 \mathrm{~cm}$-long cuvette. For these experiments we selected a representative GFP mutant, the enhanced GFP (EGFP), exhibiting a single absorption peak around $490 \mathrm{~nm}$ (associated to the anionic form of the chromophore [18]) and improved brightness after blue-light excitation with respect to wild-type GFP [5].

Figure 1 shows the steady-state absorption (dotted line) and emission (solid line) spectra of EGFP together with a schematic configuration-coordinate diagram for the anionic state. The absorption peak at $490 \mathrm{~nm}$ corresponds to the vertical electronic transition labeled as A. The emission lineshape displays two bands associated to transitions ( $\mathrm{B}$ and $\mathrm{C}$ in the diagram) from the bottom of the excited state band into the first two vibrational levels of the ground state. The lower panel of Fig. 1 shows the wavelength dependence of the average EGFP differential transmissivity $(\Delta \mathrm{T} / \mathrm{T})$ in the very first picoseconds following impulsive excitation. The observed positive values at the absorption and emission maxima can be assigned to ground-state absorption bleaching and stimulated emission from the excited 
state. In the inset of the lower panel of Fig. 1, a typical time trace with low sampling frequency of $\Delta \mathrm{T} / \mathrm{T}$ (at $500 \mathrm{~nm}$ ) is reported. The signal remains constant during the first tens of picoseconds in agreement with expected values of fluorescence lifetime $(\approx 3.3 \mathrm{~ns}$ [8]).

Superimposed to the long-lasting value of the EGFP transmissivity we detected subpicosecond oscillations during the first two picoseconds following excitation. In Fig. 2 (left side) a representative time-dependent $\Delta \mathrm{T} / \mathrm{T}$ at $530 \mathrm{~nm}$ is reported. Similar modulations were observed at other wavelengths. This oscillation pattern is a direct evidence of coherent dynamics of electronic wave packets in the GFP chromophore and yields two distinct frequencies at $497 \mathrm{~cm}^{-1}$ (period $67 \mathrm{fs}$ ) and $593 \mathrm{~cm}^{-1}$ (period $59 \mathrm{fs}$ ) with dephasing time of about 1 ps. The two peaks in the power spectrum shown in the inset of the figure are clearly distinguishable and were found at unaltered position for the wavelengths studied. The measured frequencies correspond to the energy spacing between consecutive vibronic levels of the chromophore in the ground and excited states. In our case, for pump-pulse duration significantly shorter than the oscillation period, we expect the oscillatory signal to be dominated by excited-state dynamics, since the wave packet does not move greatly from the Franck-Condon region during excitation [19]. The ground-state vibrational frequency can also be roughly extracted from the energy spacing between bands B and $\mathrm{C}$ in the emission lineshape shown in Fig. 1 . This procedure gives a value of $\approx 660 \mathrm{~cm}^{-1}$.

However, in order to unambiguously establish that the dominant mode (at $497 \mathrm{~cm}^{-1}$ ) corresponds to the GFP dynamics in the excited state, we considered the spectral dependence of the amplitude and phase of the oscillatory pattern [15]. The coherent oscillation of a vibronic wave packet in the excited state, in fact, causes periodic wavelength shifts in stimulated emission. This should yield periodic changes in the intensity of the stimulated emission with maxima where the slope of the emission lineshape is large (at 500 and 530 nm, see Fig. 1) and minima at the peak position and wings of the emission spectrum. In addition, the oscillatory pattern at wavelengths corresponding to the two sides of the emission peak must be out of phase. Similar arguments relate the vibronic wave packet in the ground state and absorption modulation to the absorption lineshape. The right side of Fig. 2 shows 
the amplitude (upper panel) and the relative phase (lower panel) of the measured transmissivity oscillations as a function of wavelength. This analysis reveals weak oscillations at wavelengths near the emission peak and at the wings, while larger on the steep sides. A strong phase change (approximately a phase inversion) centered around the wavelength of the emission maximum is observed. These data confirm that the origin of the dominant modulation at $497 \mathrm{~cm}^{-1}$ is the vibrational dynamics in the excited state. We associate the other frequency to oscillations in the ground state in light of the results of the steady-state emission lineshape analysis and of our model-chromophore calculations (see below).

On the basis of these arguments, we are now able to elucidate the vibrational pattern driven by the optical excitation process and identify the coupling mechanism of the vibrational motion to the electronic excitation. To this end we performed electronic, structural, and vibrational calculations of the isolated EGFP chromophore in the anionic state within a DFT-based ab initio molecular dynamics approach [20]. The electronic structure was calculated by using a local density exchange and correlation functional with Becke and Perdew gradient corrections [21]. Soft first-principle pseudopotential [22] were used for the interactions between valence electrons and inner cores with a 25 Ryd energy cutoff for the plane-wave basis set. Simulations with 0.15 fs time-step were performed in a $15 \AA$ cubic box, large enough to prevent interactions with the periodic images.

In the top part of Fig. 3 we show the HOMO (Highest Occupied Molecular Orbital) and LUMO (Lowest Unoccupied Molecular Orbital) of the chromophore. A charge transfer from the phenolic ring to the heterocyclic ring and a redistribution of the charge within each ring following electronic excitation is observed. This charge transfer is responsible for an increase of the proton affinity of the Tyr66 heterocyclic-ring nitrogen and may be linked to the blinking dynamics $9,23$.

The charge redistribution also causes changes in the strengths of some of the molecular bonds owing to the difference in bonding character between the ground and excited electronic states. This stimulates the dynamics seen in our experiments. The ground-state vibrational spectrum was calculated at $\mathrm{T}=300 \mathrm{~K}$ from the Fourier-transform of the veloc- 
ity autocorrelation function on a $\approx 1.5$ ps trajectory of Car-Parrinello molecular dynamics. The resulting spectrum is shown in the lower part of Fig. 3 (dashed line). The vibrational frequencies in the region above $1000 \mathrm{~cm}^{-1}$ agree within $5 \%$ with recent Raman data on the EGFP chromophore [24] and correspond to (localized or collective) stretching modes [23].

However, in thermally-equilibrated conditions not all the modes associated to the optical excitation have a significant spectral strength. We note that these modes are very unlikely to be observed in steady-state resonant Raman experiments owing to fluorescence or sample degradation 24] but are accessible within our experimental approach. In order to specifically enhance these modes, we performed runs in appropriate non-thermally-equilibrated conditions reproducing the atomic displacements induced by the electronic excitation [23]. The corresponding spectrum is shown in Fig. 3 (solid line). As expected, this simulation allows us to better identify the high-frequency streching modes in the range $1000-1650 \mathrm{~cm}^{-1}$. Remarkably, the same simulation also yields vibrations below $800 \mathrm{~cm}^{-1}$ corresponding to angular deformations of the chromophore. This fact provides evidence of intramolecular mode coupling among vibrations in two different and characteristic frequency ranges [25]. The inset of Fig. 3 reports an enlarged view in the low-frequency region of the solid-line spectrum shown in the main panel. The arrow indicates the experimentally-measured ground-state frequency $\left(593 \mathrm{~cm}^{-1}\right)$. At frequencies close to this one, we found two modes at 575 and 615 $\mathrm{cm}^{-1}$. The identification of the specific one corresponding to the experiment is beyond our accuracy. However they both correspond to collective vibrations involving angular in-plane deformation of the rings (mainly the phenolic one) and of the bridge between them. We can therefore draw unambiguous conclusions on the relevant microscopic processes involved.

The steps leading to the coupling of these low-frequency modes to the electronic photoexcitation can now be easily understood: in structures with a $\pi$-bonding system, the highfrequency stretching modes are usually directly coupled to the electronic excitation, since the primary effect of the induced electronic-density change is to shorten the single bonds and lengthen the double bonds (for example in retinals [26]). The double-ring structure of the GFP chromophore, however, introduces a strong geometric constraint that allows efficient 
coupling to the low-frequency angular modes responsible for the observed coherent dynamics. This process highlights the intramolecular coupling pathways and nuclear dynamics following photoexcitation and is a peculiar photophysical property of the GFP family.

In conclusion, we presented the coherent dynamics of single-electron vibronic wave packets following ultrafast excitation in EGFP. The analysis of coherent oscillations provided the vibrational frequencies of both the ground and excited states of the EGFP chromophore and allowed to evaluate the wave-packet dephasing time. The collective vibration excited during the optical process and its coupling mechanism to the electronic excitation have been identified by ab initio calculations.

Acknowledgements. One of us (V.T.) wishes to thank F. Buda for making available the code for Molecular Dynamics. 


\section{FIGURES}

FIG. 1. Upper panel: Enhanced green fluorescent protein (EGFP) absorption (dotted line) and fluorescence (solid line) spectra at room temperature. Letters indicate transitions between excited and ground electronic states as depicted in the schematic configuration-coordinate model. Lower panel: Wavelength dependence of the average EGFP differential transmissivity in the very first picoseconds after impulsive excitation (line is a guide to eye). Typical error bars and positions of the absorption and emission peaks are shown. The inset shows the differential transmissivity at $500 \mathrm{~nm}$ (acquired with low sampling frequency) as a function of delay between pump and probe pulses.

FIG. 2. Left side: Room-temperature enhanced green fluorescent protein (EGFP) differential transmissivity at $530 \mathrm{~nm}$ as a function of delay between pump and probe pulses. The inset shows the Fourier power spectrum of the data. Right side: Spectral dependence of the amplitude (upper panel) and relative phase (lower panel) of the measured transmissivity oscillations before damping (lines are guides to eye and typical error bars are shown).

FIG. 3. Upper side: Simulated chromophore $\left(\mathrm{C}_{10} \mathrm{O}_{2} \mathrm{~N}_{2} \mathrm{H}_{7}^{-}\right)$. The bonds with the protein backbone are cut at the level of the heterocyclic ring and saturated with hydrogen atoms. HOMO (Highest Occupied Molecular Orbital) and LUMO (Lowest Unoccupied Molecular Orbital) are represented as isocharge surfaces. Carbon, hydrogen, and nitrogen are shown in black, white, and grey, respectively. Lower side: Calculated vibrational spectra of the chromophore in thermally-equilibrated (dashed line) and non-thermally-equilibrated (solid line) conditions (see text). Inset: Enlarged view of non-thermally-equilibrated vibrational spectrum. The arrow corresponds to the experimentally-measured ground-state frequency $\left(593 \mathrm{~cm}^{-1}\right)$. 


\section{REFERENCES}

[1] M. Chalfie et al., Science 263, 802 (1994); Green Fluorescent Proteins, edited by K. F. Sullivan and S. A. Kay (Academic Press, San Diego, 1999); A. Marcello et al., Proc. Natl. Acad. Sci. USA, submitted.

[2] M. Ormö et al., Science 273, 1392 (1996).

[3] R. Heim, D. C. Prasher, and R. Y. Tsien, Proc. Natl. Acad. Sci. USA 91, 12501 (1994).

[4] R. Heim, A. B. Cubitt, and R. Y. Tsien, Nature 373, 663 (1995).

[5] G. H. Patterson et al., Biophys. J. 73, 2782 (1997).

[6] D. W. Pierce, N. Hom-Booher, and R. D. Vale, Nature 388, 338 (1997); R. M. Dickson et al., Nature 388, 355 (1997); R. A. G. Cinelli et al., Photochem. Photobiol. 71, 771 $(2000)$.

[7] P. Schwille et al., Proc. Natl. Acad. Sci. USA 97, 151 (2000).

[8] M. Chattoraj et al., Proc. Natl. Acad. Sci. USA 93, 8362 (1996).

[9] A. A. Voityuk, M. E. Michel-Beyerle, and N. Rösch, Chem. Phys. 231, 13 (1998).

[10] W. Weber et al., Proc. Natl. Acad. Sci. USA 96, 6177 (1999).

[11] M. J. Rosker, F. W. Wise, and C. L. Tang, Phys. Rev. Lett. 57, 321 (1986).

[12] T. Tokizaki et al., Phys. Rev. Lett. 67, 2701 (1991); J. Feldmann et al., Phys. Rev. Lett. 70, 3027 (1993); T. Kobayashi et al., Chem. Phys. Lett. 321, 385 (2000).

[13] M. Nisoli et al., Phys. Rev. Lett. 77, 3463 (1996).

[14] L. Zhu et al., Phys. Rev. Lett. 72, 301 (1994); M. H. Vos et al., Proc. Natl. Acad. Sci. USA 91, 12701 (1994); U. Liebl et al., Nature 401, 181 (1999).

[15] Q. Wang et al., Science 266, 422 (1994). 
[16] The term "single-electron wave packet" is used to indicate that only one electron is photoexcited in the GFP molecules and that the following dynamics of each molecule is independent of the others.

[17] G. Cerullo, M. Nisoli, and S. De Silvestri, Appl. Phys. Lett. 71, 3616 (1997).

[18] K. Brejc et al., Proc. Natl. Acad. Sci. USA 94, 2306 (1997).

[19] S. Mukamel, Principles of Nonlinear Optical Spectrosocpy (Oxford University Press, New York, 1995).

[20] R. Car and M. Parrinello, Phys. Rev. Lett. 55, 2471 (1985).

[21] J. P. Perdew and A. Zunger, Phys. Rev. B 23, 5048 (1981); A. D. Becke, Phys. Rev. A 38, 3098 (1988); J. P. Perdew, Phys. Rev. B 33, 8811 (1986).

[22] D. Vanderbilt, Phys. Rev. B 43, 7892 (1990).

[23] V. Tozzini and R. Nifosì, J. Phys. Chem. B, submitted.

[24] A. F. Bell et al., Biochemistry 39, 4423 (2000).

[25] K. Moritsugu, O. Miyashita, and A. Kidera, Phys. Rev. Lett. 85, 3970 (2000).

[26] B. Curry et al., in Advances in Infrared and Raman Spectroscopy, vol. 12, edited by R. J. H. Clark and R. E. Hester (Heyden, London, 1985). 

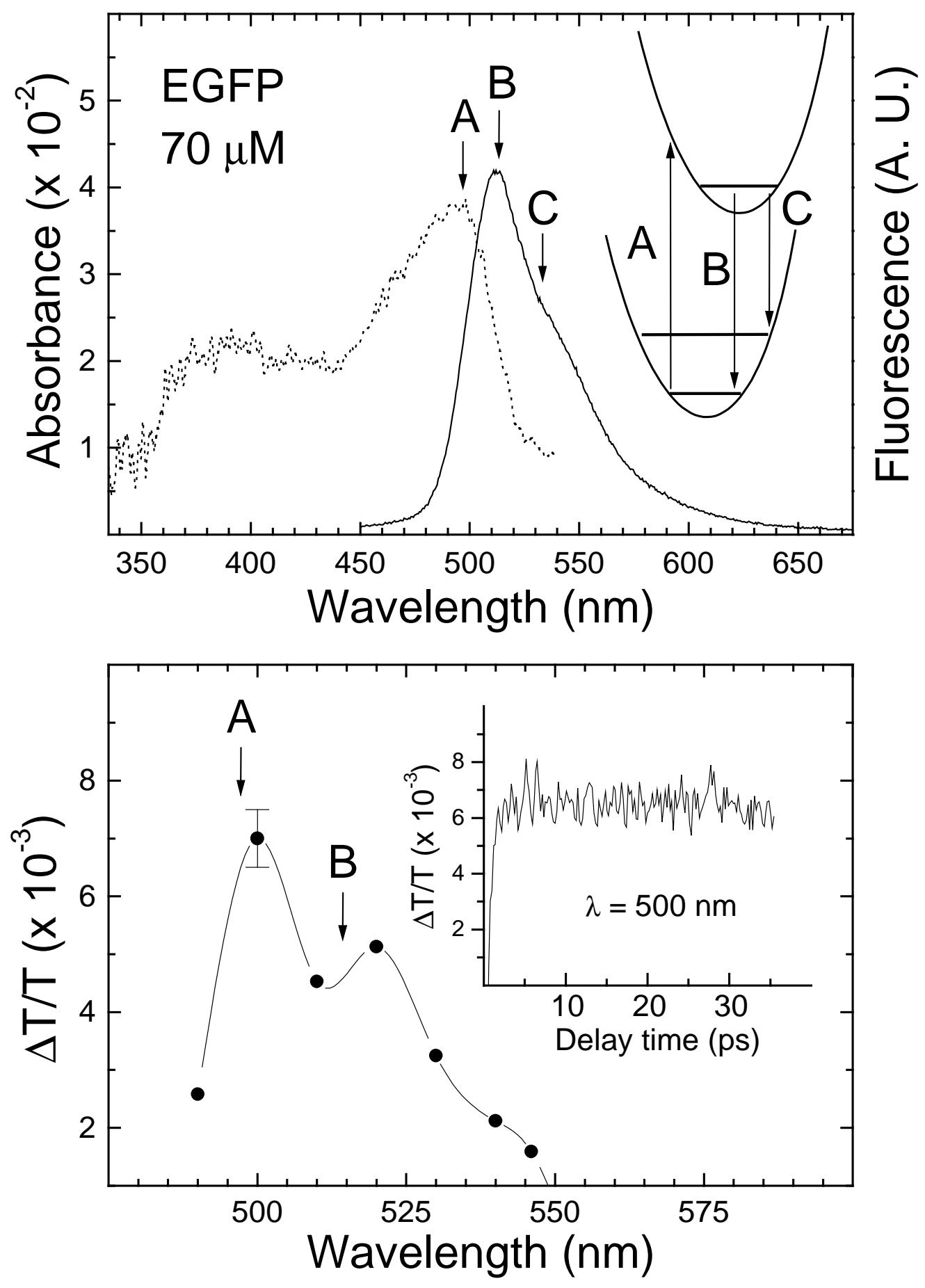


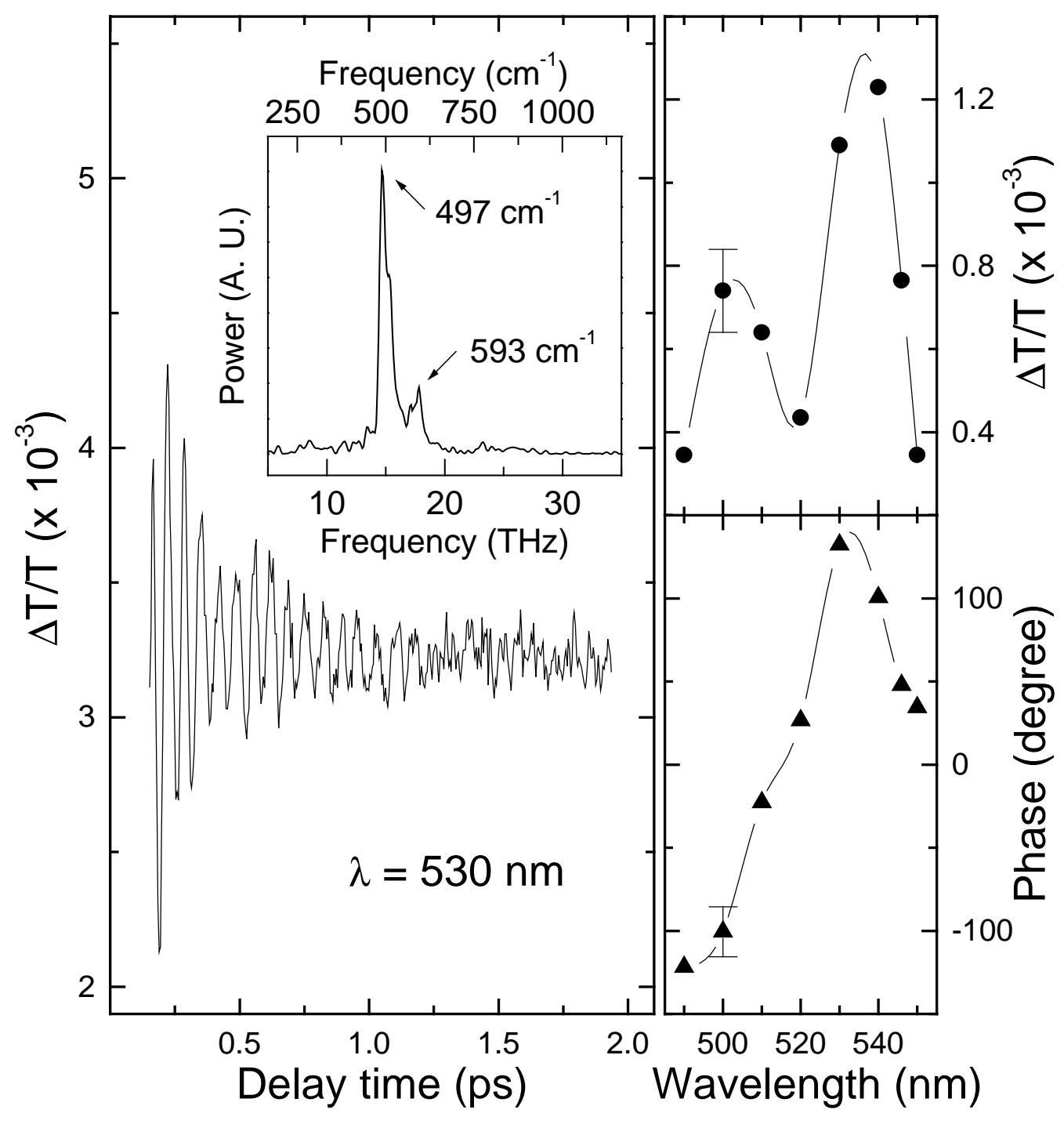



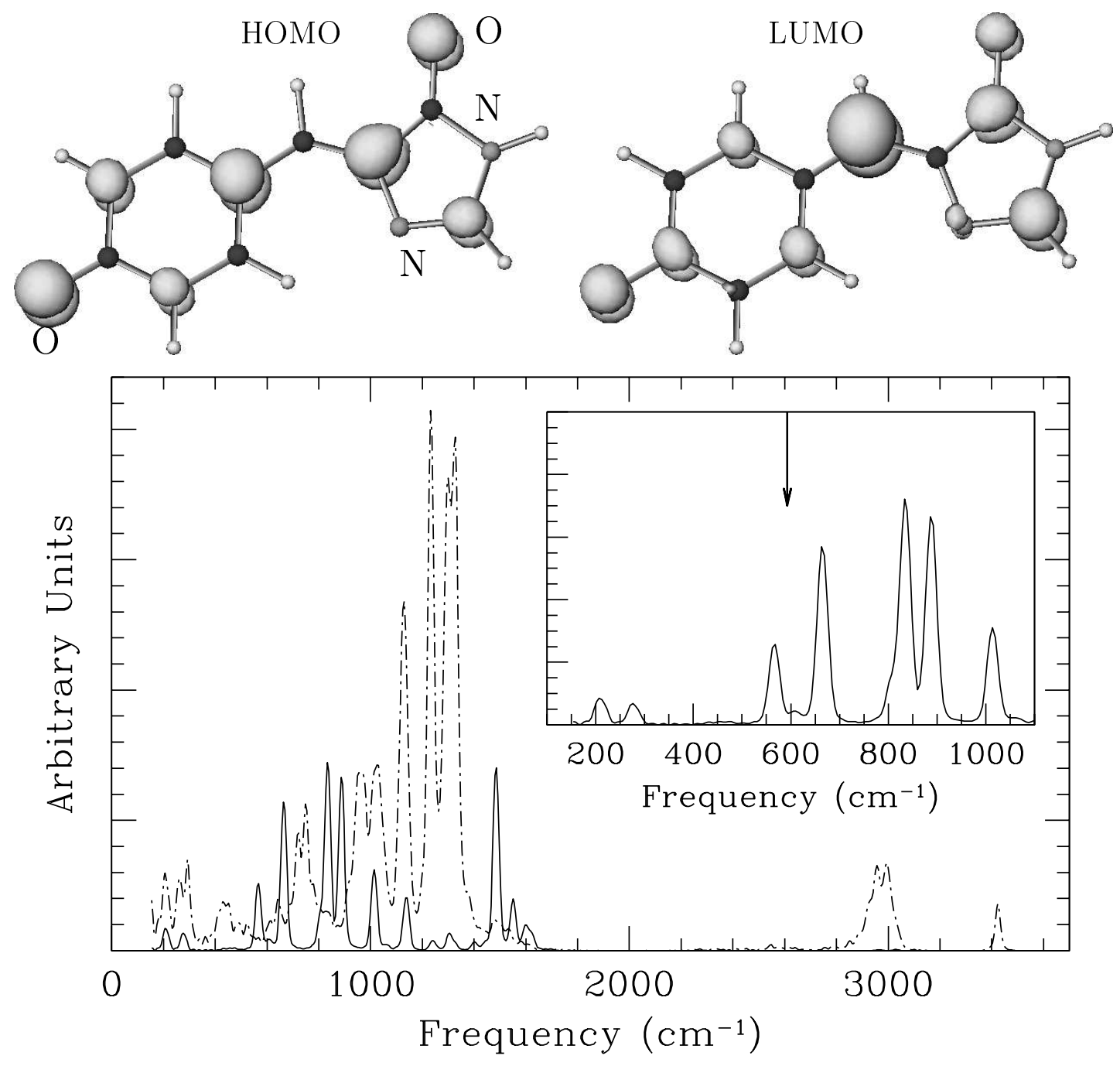